\title{
ESTUDO DAS PROPRIEDADES MAGNÉTICAS DE UM SMC INFLUENCIADAS PELO LUBRIFICANTE*
}

\author{
Silmar do Nascimento Castro ${ }^{1}$ \\ Franciele Peruchi Ronchi ${ }^{2}$ \\ Diego Pacheco Wermuth ${ }^{3}$ \\ Matheus Fortes Stiborski ${ }^{4}$ \\ Monir Göethel Borba ${ }^{5}$ \\ Lirio Schaeffer ${ }^{6}$
}

\section{Resumo}

Este estudo visa identificar as influências sofridas na mistura de diferentes porcentagens de lubrificantes com SMCs (Compósitos Magnéticos Macios). E para isso foi utilizado os conhecimentos da metalurgia do pó durante as etapas de mistura, compactação e sinterização das amostras, os resultados foram obtidos através do ensaio do anel de Rowland que possibilita a obtenção das curvas magnéticas do composto.

Palavras-chave: Metalurgia do pó; SMCs, Propriedades magnéticas.

\section{STUDY OF MAGNETIC PROPERTIES OF A SMC INFLUENCED BY LUBRICANT}

\begin{abstract}
IThis study aims to identify the influences suffered in mixing different percentages of lubricants SMCs (Soft Magnetic Composites). The powder metallurgy knowledge was used for mixing, compaction and sintering of the samples, were obtained by Rowland ring test which resulting in magnetic curves.
\end{abstract}

Keywords: Powder metallurgy; SMCs; Magnetic properties.

1 Graduando em Engenharia Metalúrgica na Universidade Federal do Rio Grande do Sul, Bolsista de Iniciação Tecnológica do Laboratório de Transformação Mecânica (LdTM), Porto Alegre, RS, Brasil.

2 Mestre em Engenharia de Minas, Metalúrgica e Materiais (PPGE3M). Laboratório de Transformação Mecânica. (LdTM), Depto. de Metalurgia, UFRGS, Porto Alegre, RS, Brasil. Professora na Faculdade SATC, Criciúma, SC, Brasil.

3 Doutorando do Programa de Pós-Graduação em Engenharia de Minas, Metalúrgica e Materiais (PPGE3M). Laboratório de Transformação Mecânica. (LdTM), Depto. de Metalurgia, UFRGS, Porto Alegre, RS, Brasil.

4 Graduando em Engenharia Metalúrgica na Universidade Federal do Rio Grande do Sul, Bolsista de Iniciação Tecnológica do Laboratório de Transformação Mecânica (LdTM), Porto Alegre, RS, Brasil.

5 Mestrando do Programa de Pós-Graduação em Engenharia de Minas, Metalúrgica e Materiais (PPGE3M). Laboratório de Transformação Mecânica. (LdTM), Depto. de Metalurgia, UFRGS, Porto Alegre, RS, Brasil.

6 Engenheiro Mecânico, Doutor em Engenharia, Professor na Universidade Federal do Rio Grande do Sul, Diretor do Laboratório de Transformação Mecânica (LdTM), Porto Alegre, RS, Brasil. 


\section{INTRODUÇÃO}

\subsection{Metalurgia do Pó}

A Metalurgia do Pó é um processo de fabricação que produz peças metálicas através da sinterização do pó metálico, o processo se distingue por manter a temperatura abaixo da temperatura de fusão do elemento principal do compósito.

As principais etapas que constituem a metalurgia do pó são:

- Coleta da matéria prima, no caso pós-metálicos.

- Mistura dos pós, fase onde pode se adicionar lubrificantes ou não e outros elementos afim da obtenção da liga.

- Compactação, etapa onde se aplica uma determinada pressão na liga obtida na fase anterior, com intuito de moldar o material de acordo com sua matriz de compactação.

- Cura ou Sinterização, etapa onde é feito o tratamento térmico do material compactado, isso é necessário para conferir ao material maior resistência mecânica.

- Caracterização, fase onde se avalia as perdas ou ganhos de densidade, massa e dimensões do material. No termino do processo obtemos o produto acabado, onde o material está pronto para ser utilizado em sua devida aplicação o esquema abaixo mostra as etapas da metalurgia do pó (Figura 1). [1].



Figura 1. Etapas da Metalurgia do Pó. [1] 


\subsection{SMCs (Compósitos Magnéticos Macios)}

Compósitos Magnéticos Macios ou SMCs como são chamados, são partículas de pó de ferro microencapsuladas cobertas por uma camada isolante, como mostra a figura 2. A finalidade dessa camada é unir as partículas e também serve como isolante elétrico, fazendo com que o material possa ser utilizado em futuras aplicações eletromagnéticas. ${ }^{[1,2,3]}$

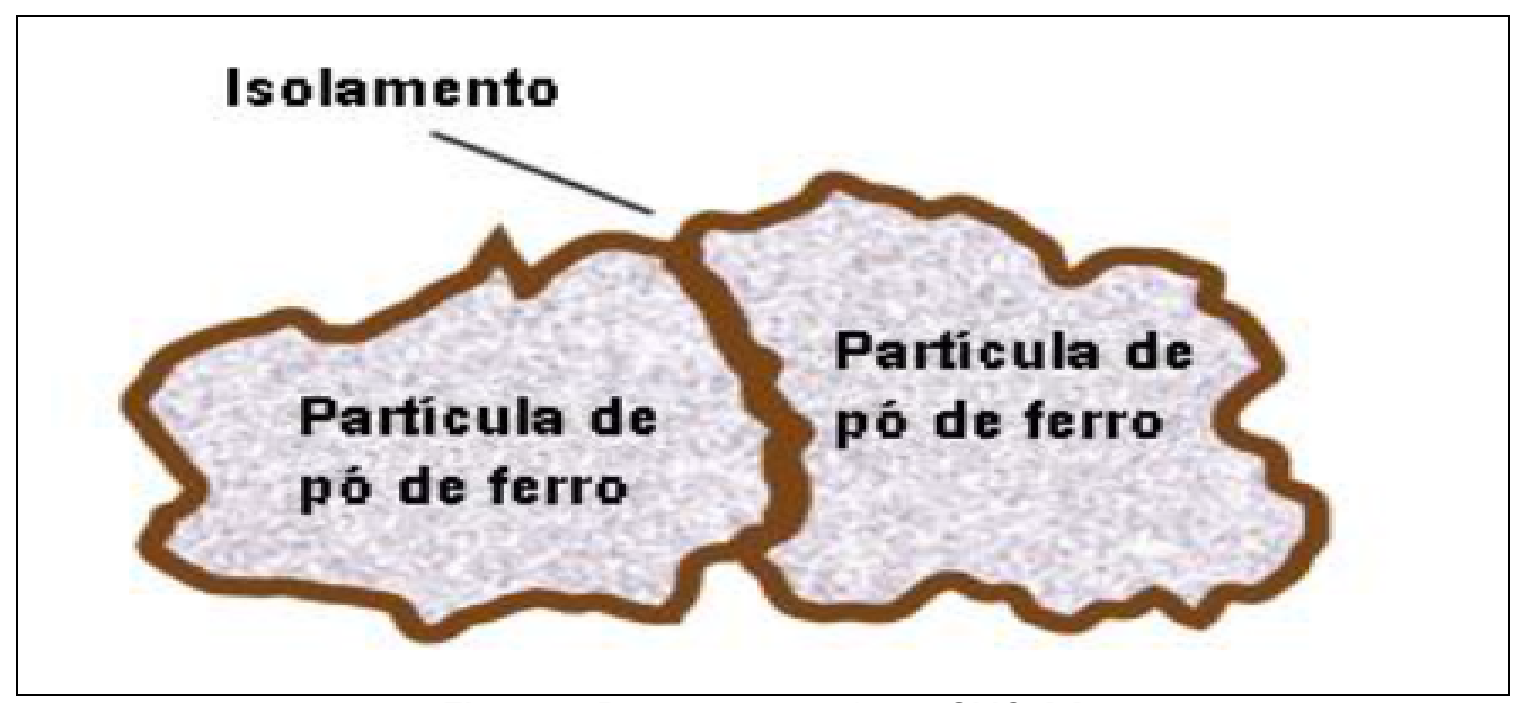

Figura 2. Representação de um SMC. [2]

\subsection{Anel de Rowland}

O teste do anel de Rowland foi desenvolvido originalmente por Henry A.Rowland (1848-1901) seu custo de fabricação foi baixo, considerando os parâmetros da época.

O Anel de Rowland é coberto uniformemente por uma bobina magnetizadora e uma sensora, que fica concentrada em um polo do anel, medindo o fluxo magnético produzido no centro do anel. (Figura 3)

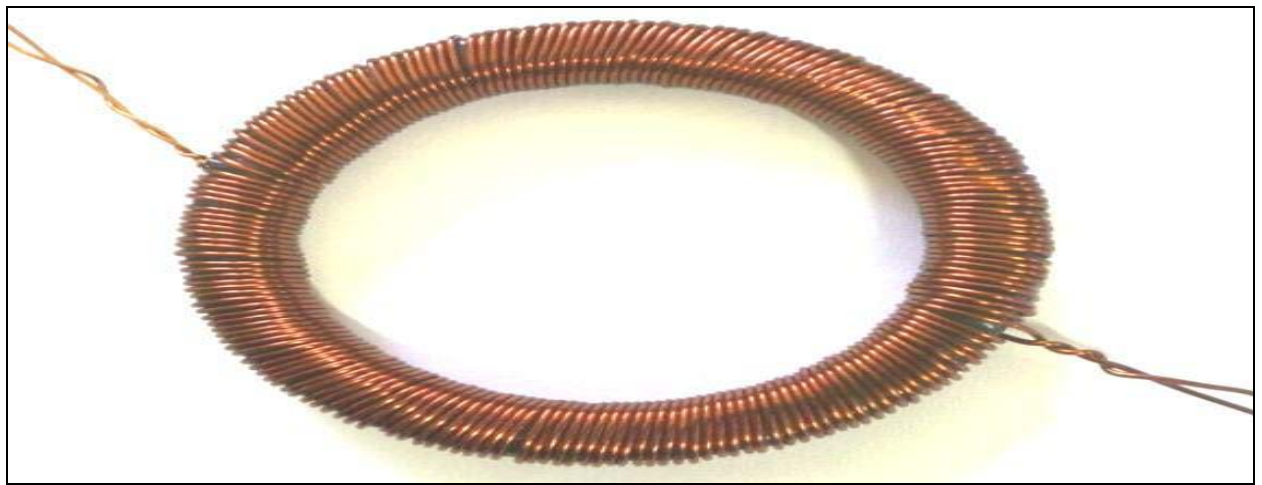

Figura 3. Anel de Rowland bobinado e propor para o ensaio. 


\subsection{Propriedades Magnéticas}

\subsubsection{Curva de histerese}

Quando uma bobina, com um determinado material em seu núcleo é submetida a uma corrente elétrica I, será gerado no interior desta bobina um campo magnético $\mathrm{H}$ e a peça sofrerá uma indução magnética $B$. Se o material do núcleo for ferromagnético, linhas de fluxo serão induzidas em seu interior, resultando em uma curva de relação BxH. Esta curva é chamada de curva de histerese ou ciclo de histerese, demonstrada na figura 4. ${ }^{[4]}$

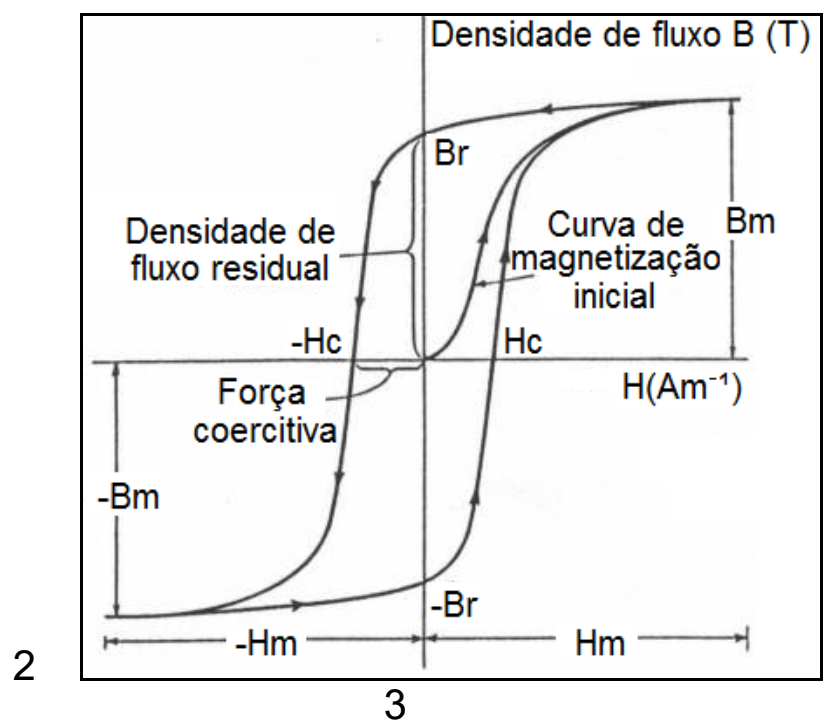

Figura 4. Ciclo de histerese de um material magnético macio para diferentes valores de indução magnética. [5]

Desta forma, denomina-se magnetismo remanente ou retentividade $B$ o ponto em que a curva corta o eixo $B$, no quadrante superior e representa a indução magnética residual que permanece no material sem campo magnético aplicado $(\mathrm{H}=0)$. $E$ denomina-se força coercitiva ou coercitividade $\mathrm{H}_{c}$ o ponto em que a curva corta o eixo de $\mathrm{H}$ no quadrante esquerdo e representa o campo magnético necessário para desmagnetizar o espécime $(B=0)$. A permeabilidade magnética $\mu$ está relacionada com o ponto de maior declividade da curva de histerese. A indução de saturação $B_{\max }$ pode ser visualizada a partir dos valores de campo onde a indução tende a permanecer constante ou com declividade quase nula ${ }^{[6]}$.

\subsubsection{Curva de Magnetização}

A fonte da magnetização espontânea está relacionada a uma propriedade atômica das partículas chamada spin. Entre a escala atômica do spin do elétron e magnetização macroscópica encontra-se outra subestrutura chamada domínios [6].

Abaixo da temperatura Curie, spins de elétrons de átomos vizinhos se alinham aleatória e espontaneamente e cada região alinhada é chamada de domínio. Quando um campo é aplicado, os domínios alinhados podem crescer, criando uma magnetização. ${ }^{[7]}$

Ao submeter um material ferromagnético, que inicialmente foi desmagnetizado a um aumento contínuo de campo magnetizante $\mathrm{H}$, a relação entre $\mathrm{H}$ e densidade de campo B é mostrada pela curva de magnetização (figura 5). ${ }^{[8]}$ 
Com o campo $\mathrm{H}$ aplicado, os domínios mudam de forma e tamanho pelo movimento dos limites do domínio. As estruturas esquemáticas do domínio são representadas na figura 5 , de $U$ até $Z .{ }^{[7]}$

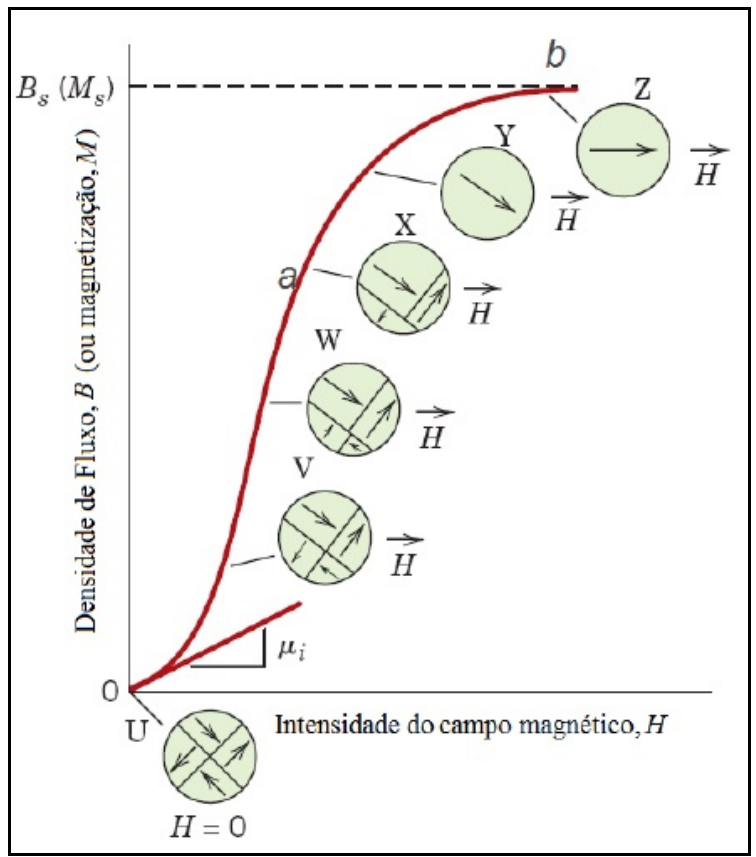

Figura 5. Curva de magnetização B-H de um material ferromagnético desmagnetizado. Configurações de domínio durante várias fases da magnetização são representados. Densidade do fluxo na saturação Bs, Magnetização Ms, e permeabilidade inicial $\mu$ i. [7]

Da curva de magnetização pode-se extrair informações importantes para a avaliação de materiais para aplicações magnéticas, como: Saturação Máxima e Permeabilidade. [4]

\subsubsection{Permeabilidade magnética $(\mu)$}

O acréscimo nos valores de permeabilidade magnética das ligas provoca um menor campo magnético no núcleo e um maior campo no entreferro. Para uma máquina elétrica rotativa, caso dos motores e geradores, o espaço compreendido entre o núcleo do rotor e o estator, deve apresentar o maior campo magnético possível, acarretando assim uma maior indução magnética ${ }^{[8]}$.

\subsubsection{Indução de Saturação ( $\left.B_{\max }\right)$}

A saturação magnética limita a maior indução possível no material. Nos núcleos de máquinas elétricas, quanto maior a indução de saturação, maior será o fluxo de entreferro ${ }^{[4]}$.

\section{MATERIAIS E MÉTODOS}

Para fazer os devidos testes foi utilizado o pó 700 1P da família Somaloy e como lubrificantes foi utilizado Kenolube nas porcentagens com relação ao pó de $0,2 \%$, $0,4 \%$ e $0,6 \%$. Ambos os matérias são produzidos e distribuídos pela empresa Höganäs.

Foi feito a divisão do pó puro em 3 amostras iguais e em seguida adicionado quantidade suficiente de lubrificante de acordo com a porcentagem estudada, os 
componentes foram misturados durante uma hora e após isso estavam prontos para etapa de compactação do composto.

$\mathrm{Na}$ etapa de compactação foi utilizada matriz que fizesse com que o corpo de prova fosse gerado na forma de anel (Figura 6).

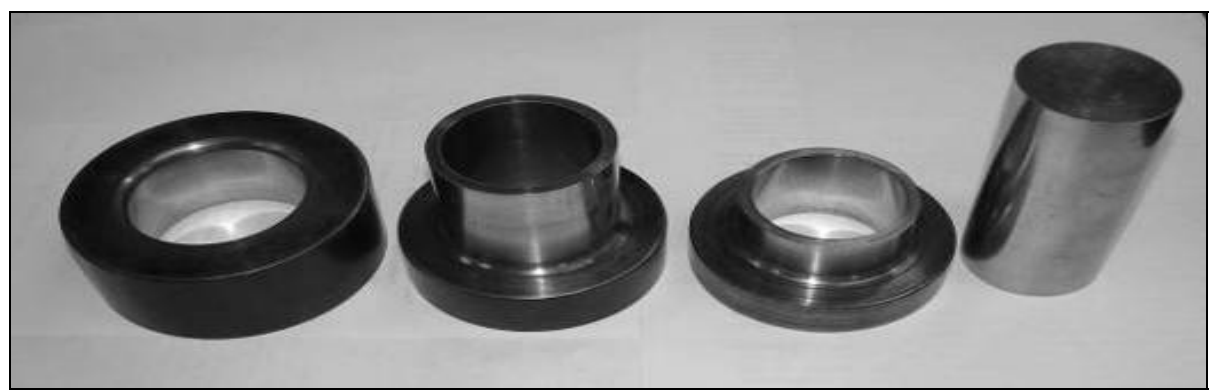

Figura 6. Matriz para compactação dos corpos de prova na forma de anel.

Foram compactadas três amostras de cada material em formato de anel de acordo com as matrizes, com forças correspondente de 800mpa. (Figura 7).

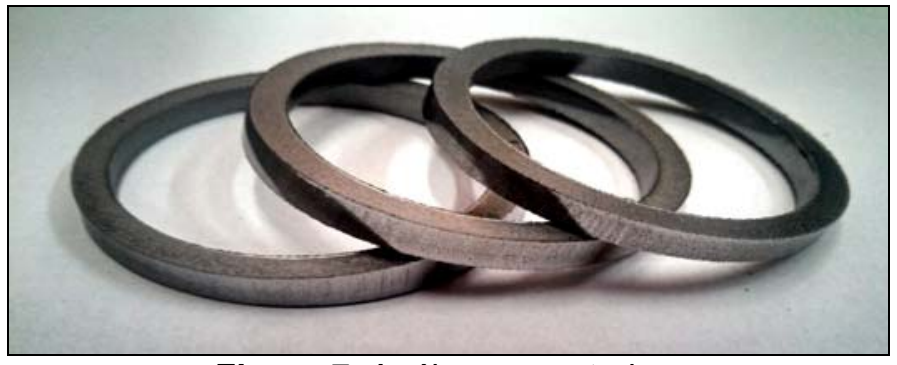

Figura 7. Anéis compactados.

Para a compactação das amostras foi utilizada a máquina universal de ensaio EMIC que possui capacidade de $600 \mathrm{kN}$ e opera entre as faixas de velocidade de 0,01 a $300 \mathrm{~mm} / \mathrm{min}$. (Figura 8).

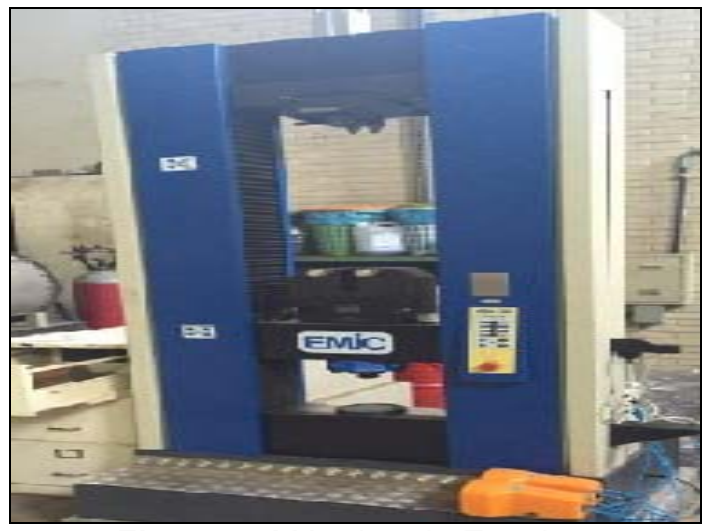

Figura 8. Máquina de Ensaios.

As amostras foram sinterizadas durante 30 minutos a uma temperatura de $530^{\circ} \mathrm{C}$, a atmosfera utilizada foi ar-teorico e para obtenção das curvas de histeres e magnetização foram desenvolvidos os anéis de Roland (Figura 9).

Para fazer os anéis utilizou-se fita isolante e fio de cobre AWG $26(0,41 \mathrm{~mm}$ de diâmetro) para o bobinamento primário e novamente fita isolante e fio de cobre AWG $23(0,57 \mathrm{~mm}$ de diâmetro) para o bobinamento secundário. 


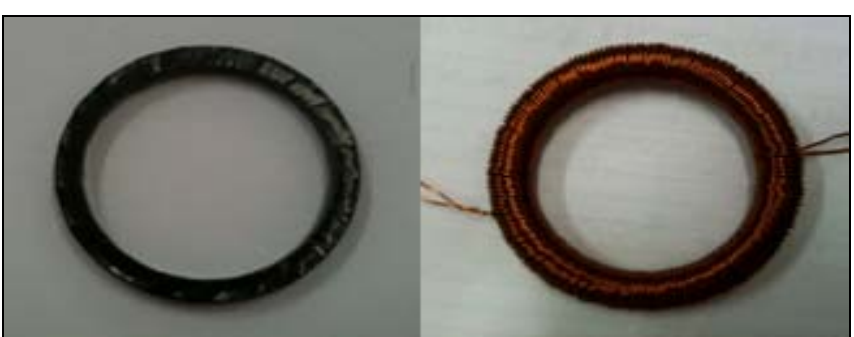

Figura 9. Anéis de Rowland.

Após a confecção dos anéis, estes foram conectados ao equipamento RemagraphRemacomp C-710, da marca Magnet-Physik Dr. Steingroever GmbH (Figura 10) utilizado para aquisição da curva de magnetização e curva de histerese das amostras.



Figura 10. Equipamento Remagraph-Remacomp C-710.

\section{RESULTADOS E DISCUSSÃO}

As curvas obtidas foram típicas de materiais magnéticos macios, e com esse ensaio foi possível à retirada de dados magnéticos importantes dos materiais como a indução de saturação, magnetismo remanente, força coercitiva, as perdas por histerese e a permeabilidade máxima. A indução de saturação ou indução máxima pode ser visualizada a partir dos valores de campo onde a indução tende a permanecer constante ou com declividade quase nula

Nas figuras 11 e 12, pode-se visualizar as curvas resultantes do material Somaloy 7001P.

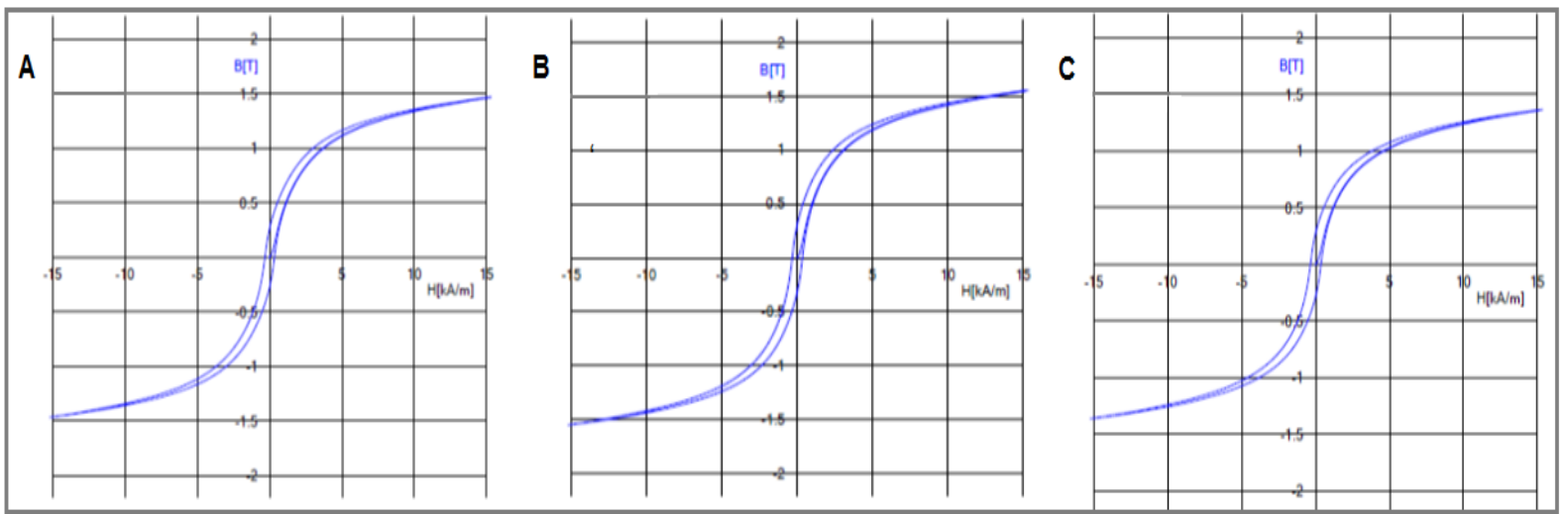

Figura 11. Curva de histerese Somaloy, $7001 \mathrm{P}+0,2 \%$ Lubrificante $(\mathrm{A}), 7001 \mathrm{P}+0,4 \%$ Lubrificante (B) e $7001 \mathrm{P}+0,6 \%$ Lubrificante (C). 


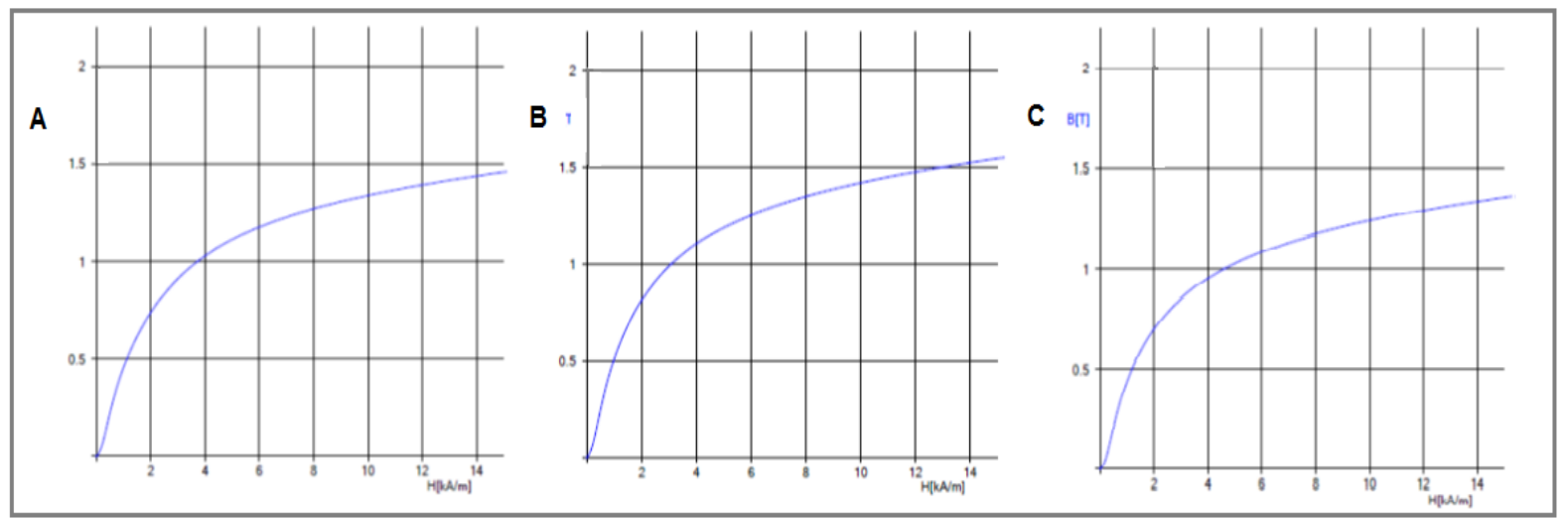

Figura 12. Curva de Magnetização Somaloy, $7001 P+0,2 \%$ Lubrificante (A), $7001 P+0,4 \%$ Lubrificante (B) e $7001 \mathrm{P}+0,6 \%$ Lubrificante (C).

Os resultados obtidos neste teste podem ser visualizados na tabela 1.

Tabela 1. Propriedades Magnéticas dos corpos de prova na forma de anel

\begin{tabular}{|l|c|c|c|}
\hline & $\begin{array}{c}\text { Somaloy 700 1P } \\
+\mathbf{0 , 2 \%} \\
\text { Lubrificante }\end{array}$ & $\begin{array}{c}\text { Somaloy 700 1P } \\
+\mathbf{0 , 4 \%} \\
\text { Lubrificante }\end{array}$ & $\begin{array}{c}\text { Somaloy 700 1P } \\
+\mathbf{0 , 6 \%} \\
\text { Lubrificante }\end{array}$ \\
\hline Indução de Saturação (T) & 1,45 & 1,54 & 1,37 \\
\hline Magnetismo remanente (T) & 0,269 & 0,312 & 0,281 \\
\hline Força coercitiva (KA/m) & 0,328 & 0,337 & 0,354 \\
\hline Perdas por histerese (kJ/m $\left.\mathbf{m}^{\mathbf{3}}\right)$ & 1,9 & 2 & 1,9 \\
\hline Permeabilidade Máxima & 379 & 424 & 366 \\
\hline
\end{tabular}

Foi verificado nos resultados obtidos um aumento da Indução de saturação quando inserido $0,4 \%$ de lubrificante comparado com a adição de $0,2 \%$ de lubrificante. Quando inseridos $0,6 \%$ de lubrificante a indução de saturação sofre um decaimento. Os resultados para a força coercitiva e perdas por histerese permanecem muito proximos um dos outros entre os tres casos.

O material que foi adicionado $0,4 \%$ de lubrificante tambem obteve maior permeabilidade magnetica.

\section{CONCLUSÃO}

Através dos testes feitos neste estudo utilizando o material Somaloy 700 1P, verificou-se que o acréscimo do lubrificante ao SMC acarreta algumas alterações nas propriedades magnéticas do compósito. Visando a utilização em motores elétricos, O SMC com adição de $0,4 \%$ de lubrificante atingiu maior indução de saturação e permeabilidade magnética, nos demais aspectos as matérias alcançaram resultados semelhantes.

Sendo assim foi possível concluir através deste trabalho que a adição de lubrificante no SMC influencia suas propriedades magnéticas e o processamento do material deve ser feito de acordo com o objetivo final de sua utilização. 


\section{Agradecimentos}

À Universidade Federal do Rio Grande do Sul, principalmente ao laboratório de transformação mecânica (ldtm) pela disponibilização dos recursos materiais e tecnológicos utilizados na elaboração deste trabalho. Aos órgãos CAPES e CNPq pelo apoio a projetos e investimento na área da pesquisa. Ás empresas hoganas e imobras motores elétricos pelo investimento nessa pesquisa.

\section{REFERÊNCIAS}

1 GRUPO SETORIAL DE METALURGIA DO PÓ. A metalurgia do pó: Alternativa Econômica com Menor Impacto Ambiental São Paulo: Editora Metallun Eventos Técnicos. 2009.

2 HULTMAN L.O.; JACK A.G. Soft magnetic composites - motor design issues and applications. Proceedings of the International Conference on Powder Metallurgy and Particulate Materials. 2004.

3 JANSSON, P. Processing Aspects of Soft Magnetic Composites. Presented at EURO PM2000 in Munich, Germany on October, 2000.

4 BARBOZA, Juliano Soares. Caracterização de Compósitos Magnéticos Macios Desenvolvidos Através da Matalurgia do Pó Aplicados a Núcleos de Máquinas Elétricas. Dissertação. PPGE3M: UFRGS, 2009.

5 KRAUS, J.D.; CARVER, K.R. Eletromagnetismo. Rio de Janeiro: Guanabara Dois, 1978. $780 \mathrm{p}$.

6 JILES, D. Introduction to Magnetism and Magnetic Materials. 2 ed. Boca Raton: Chapman and Hall, 1998.

7 LUNA, Hilbert Harold. Desenvolvimento de Ímãs de Nd-Fe-B Aplicados em Máquinas Elétricas (Motores). Tese. PPGE3M: UFRGS, 2012.

8 CALLISTER W. D. Ciência e Engenharia de Materiais - Uma Introdução. 7 ed. Rio de Janeiro: LTC, 2008. 